\title{
Dissociable Effects of Cocaine Dependence on Reward Processes: The Role of Acute Cocaine and Craving
}

\author{
Emma Jane Rose ${ }^{*, 1,2}$, Betty Jo Salmeron', Thomas J Ross', James Waltz ${ }^{3}$, Julie B Schweitzer ${ }^{4}$ and \\ Elliot A Stein'
}

'Neuroimaging Research Branch, National Institute on Drug Abuse - Intramural Research Program, NIH, Baltimore, MD, USA; ${ }^{2}$ Program for Translational Research on Adversity and Neurodevelopment, Bennett Pierce Prevention Research Center, Health and Human Development, The Pennsylvania State University, University Park, PA, USA; ${ }^{3}$ Maryland Psychiatric Research Center, University of Maryland School of Medicine, Baltimore, MD, USA; ${ }^{4}$ MIND Institute, Department of Psychiatry and Behavioral Sciences, University of Califomia Davis School of Medicine, Sacramento, CA, USA

\begin{abstract}
The relative impact of chronic vs acute cocaine on dependence-related variability in reward processing in cocaine-dependent individuals (CD) is not well understood, despite the relevance of such effects to long-term outcomes. To dissociate these effects, $C D(N=15)$ and healthy controls $(\mathrm{HC} ; \mathrm{N}=15)$ underwent $\mathrm{MRI}$ two times while performing a monetary incentive delay task. Both scans were identical across subjects/groups, except that, in a single-blind, counterbalanced design, CD received intravenous cocaine (30 mg/70 kg) before one session (CD+cocaine) and saline in another (CD+saline). Imaging analyses focused on activity related to anticipatory valence (gain/loss), anticipatory magnitude (small/medium/large), and reinforcing outcomes (successful/unsuccessful). Drug condition (cocaine vs saline) and group ( $\mathrm{HC}$ vs CD+cocaine or CD+saline) did not influence valence-related activity. However, compared with $\mathrm{HC}$, magnitude-related activity for gains was reduced in CD in the left cingulate gyrus post-cocaine and in the left putamen in the abstinence/saline condition. In contrast, magnitude-dependent activity for losses increased in $\mathrm{CD}$ vs $\mathrm{HC}$ in the right inferior parietal lobe post-cocaine and in the left superior frontal gyrus post-saline. Across outcomes (ie, successful and unsuccessful) activity in the right habenula decreased in CD in the abstinence/saline condition vs acute cocaine and HC. Cocaine-dependent variability in outcome- and loss-magnitude activity correlated negatively with ratings of cocaine craving and positively with how high subjects felt during the scanning session. Collectively, these data suggest dissociable effects of acute cocaine on non-drug reward processes, with cocaine consumption partially ameliorating dependencerelated insensitivity to reinforcing outcomes/'liking', but having no discernible effect on dependence-related alterations in incentive salience/ 'wanting'. The relationship of drug-related affective sequelae to non-drug reward processing suggests that CD experience a general alteration of reward function and may be motivated to continue using cocaine for reasons beyond desired drug-related effects. This may have implications for individual differences in treatment efficacy for approaches that rely on reinforcement strategies (eg, contingency management) and for the long-term success of treatment.

Neuropsychopharmacology (2017) 42, 736-747; doi:I0.1038/npp.2016.161; published online 5 October 2016
\end{abstract}

\section{INTRODUCTION}

Approximately $14 \%$ of Americans aged 12 years and over report lifetime cocaine use, with $\sim 1$ million individuals experiencing cocaine use disorder/dependence (CUD) in the United States (SAMHSA, 2014). Cocaine use is associated with high personal, social, and economic burden and high post-treatment recidivism rates. Thus, the mechanisms underlying cocaine abuse/dependence, especially those that are clinically relevant, urgently need to be determined.

* Correspondence: Dr EJ Rose, Program for Translational Research on Adversity and Neurodevelopment, Bennett Pierce Prevention Research Center, Health and Human Development, The Pennsylvania State University, 308C Biobehavioral Health, University Park, PA I6802, USA, Tel: + | 8|4865 0| |4, Fax: + | 8 |4 865 2580,

E-mail: emmajanerose@psu.edu

Received 8 February 20 16; revised 19 July 20 I6; accepted 22 July 2016 ; accepted article preview online 19 August 2016
Since motivational changes underlie key aspects of addiction (eg, craving, intoxication/bingeing, and withdrawal/ anhedonia) (Volkow et al, 2011), drug dependence can be characterized as a disease of altered reward/motivation (Volkow et al, 2010). Addiction-related motivational abnormalities are mediated by functional alterations in mesocorticolimbic (MCL) and nigrostriatal (NS) dopamine (DA) regions that constitute the brain's reward network (Diekhof et al, 2008). These regions support a diverse range of reward-related functions, including incentive salience (ie, 'wanting') (Knutson et al, 2001, 2003), the hedonic experience of reward (ie, 'liking') (O'Doherty et al, 2001), and reward learning (Asaad and Eskandar, 2011; Berns et al, 2001; McClure et al, 2003; Schultz, 1998,2000). Although less commonly considered in human studies of reward, the lateral habenula $(\mathrm{LHb})$ has an important role in modulating reward-related activity in DA neurons (Matsumoto and Hikosaka, 2008) and is ideally positioned to mediate 
interactions between reward-related and associated brain networks (Hikosaka et al, 2008).

The brain's functional response to acute cocaine involves the same reward pathways that mediate acute drug responses in preclinical models (Breiter et al, 1997; Kufahl et al, 2005; Risinger et al, 2005), with large-scale brain networks that involve MCL and NS regions found to be dysfunctional in those who abuse cocaine ( $\mathrm{Gu}$ et al, 2010; Tomasi et al, 2010). We recently observed that reward learning for a natural reinforcer (juice) was dysregulated in cocaine-dependent individuals (CD). Non-treatment-seeking CD exhibited increased sensitivity to unexpected negative outcomes and decreased responsiveness to positive/rewarding outcomes in a distributed network of regions, including those along dopaminergic pathways (Rose et al, 2014). Studies using secondary reinforcers (ie, money) also suggest cocaine-specific effects. Current cocaine misuse predicts reduced sensitivity to rewarding monetary outcomes, an effect mediated by dysfunction in prefrontal reward regions that is most pronounced in recent abstinence (Goldstein et al, 2007a, b, 2008; Parvaz et al, 2012). In addition, anticipation for and receipt of monetary rewards leads to greater activation in CD vs healthy controls $(\mathrm{HC})$ in several reward regions (eg, ventral striatum and caudate) (Jia et al, 2011). Indeed, current and former users can be distinguished based on anticipatory activity in prefrontal regions (eg, anterior cingulate (ACC)) and activity for losing outcomes in the VTA, with differences in outcome-related function being related to elevated impulsivity in $\mathrm{CD}$, regardless of use status (Patel et al, 2013).

Collectively, these studies suggest compromised reward networks/regions in $\mathrm{CD}$ for primary and secondary nondrug reinforcers, which is modulated by the recency of use. This is highly relevant since cocaine-mediated changes in reward processing likely contribute to continued cocaine consumption and, in turn, high recidivism rates. Moreover, nonspecific cocaine-mediated aberrations in reward-related

Table I Participant Demographics

\begin{tabular}{|c|c|c|}
\hline & $C D(N=15)$ & $\mathrm{HC}(N=15)$ \\
\hline Age (years: mean (SD)) & $42.47(2.39)$ & $40.60(3.42)$ \\
\hline Gender (male : female) & $13: 2$ & $14: 1$ \\
\hline IQ (WASI: mean (SD)) & $97.79(9.04)$ & I04.53 (13.37) \\
\hline Education (years: mean (SD)) & $13.00(1.73)$ & | $3.53(2.67)$ \\
\hline $\begin{array}{l}\text { Race (African } \\
\text { American : Caucasian) }\end{array}$ & $14: \mid$ & $11: 4$ \\
\hline $\begin{array}{l}\text { Years of regular cocaine use } \\
\text { (mean (SD); range) }\end{array}$ & $16.20(5.87) ; 3-25$ & N/A \\
\hline $\begin{array}{l}\text { No. of days using cocaine per } \\
\text { week (mean (SD); range) }\end{array}$ & $2.23(1.57) ; 1-7$ & N/A \\
\hline $\begin{array}{l}\text { No. of rocks of cocaine used in the } \\
7 \text { days preceding study (mean } \\
\text { (SD); range) }\end{array}$ & $10.57(12.14) ; 0-40$ & N/A \\
\hline $\begin{array}{l}\text { Dollars spent on cocaine in the } \\
7 \text { days preceding the study (mean } \\
\text { (SD); range) }\end{array}$ & I 87.33 (252.23); 0-1000 & N/A \\
\hline
\end{tabular}

Abbreviations: CD, cocaine-dependent individual; $H C$, healthy control; $I Q$, intelligence quotient; N/A, not available; WASI, Wechsler Abbreviated Scale of Intelligence.

Note: All between-group comparisons (ie, $t$ or $\chi^{2}$ ) were nonsignificant. function that extend to non-drug reinforcers may impact cessation attempts, particularly when treatment strategies rely on reinforcement. What is not yet apparent is the impact that acute cocaine has on these processes and the key phenotypic characteristics of CUD that predict short- and long-term outcomes (eg, craving).

Here we considered neuronal mechanisms related to the anticipation and experience of rewarding and punishing monetary outcomes in non-treatment-seeking CD during acute abstinence (ie, $<24 \mathrm{~h}$ ) and following acute cocaine. This within-subjects comparison allowed us to consider relative functional alterations in reward processing related to the trait of cocaine dependence $v s$ the state of acute cocaine intoxication. To address issues relating to the long-term impact of cocaine dependence and relative 'normalization' of reward processing following acute administration, we included a between-subjects element in our design and compared $\mathrm{CD}$ in each condition (ie, acute abstinence and acute cocaine) to matched HC. We hypothesized that: (1) compared with HC, recently abstinent CD would exhibit a relative reduction in brain activity in regions subserving reward anticipation and outcome (eg, substantia nigra (SN), VTA, habenula, striatum, medial prefrontal cortex (mPFC)) $\left(\mathrm{H}_{1}\right)$; and (2) acute cocaine would stimulate DA pathway regions $\left(\mathrm{H}_{2 \mathrm{~A}}\right)$ and effectively 'normalize' $\left(\mathrm{H}_{2 \mathrm{~B}}\right)$ rewardrelated activity in MCL and NS pathway regions.

\section{MATERIALS AND METHODS}

\section{Participants}

HC $(N=26)$ and non-treatment-seeking $\mathrm{CD}(N=22$; see Supplementary Table S1 for intake cocaine use parameters) were recruited from the general population. Please note that CD were offered access to drug treatment services as a participation alternative. Those expressing a preference for treatment were automatically excluded. Participants were right handed, aged 18-45 years, and had no current or past DSMIV-TR Axis I or II diagnoses, except nicotine dependence and current CUD (CD only). Seven CD were removed for data quality issues (primarily excessive head motion; $>3 \mathrm{~mm}$ or $3^{\circ}$ ), resulting in an analysis cohort of $N=15$. A subsample of $15 \mathrm{HC}$ from those that passed data quality control $(N=24)$ were matched to $\mathrm{CD}$ for age, sex, race, and IQ (Table 1).

\section{Characterization}

Participants completed characterization measures including indices of psychiatric history, personality, stressful life events, and cognitive function (see Supplementary Methods for description and Supplementary Table S2 for score summaries and comparisons). CD provided a detailed cocaine use history and completed prestudy craving and withdrawal measures.

\section{Procedure}

The NIDA-IRP IRB approved this study and subjects provided pre-study written, informed consent. Participants completed training in a mock scanner and two MRI sessions. CD also completed a 'drug toleration' session before scanning (see Supplementary Methods for full description). 
HC completed experimental sessions on separate days, scheduled as closely as possible. CD were tested on consecutive days and stayed overnight between sessions (Supplementary Figure S1). MRI sessions were identical, except that, using a within-subject, single-blind, randomly counterbalanced design, $C D$ received intravenous cocaine (CD+cocaine) or saline (CD+saline) during scanning $(N=7$ cocaine first). Drug administration procedures and physiological monitoring for scanning were as described for the toleration session, that is, two, $3 \mathrm{~min} / 10 \mathrm{ml}$ injections of $30 \mathrm{mg}$ cocaine hydrochloride $/ 70 \mathrm{~kg}$ bodyweight; one injection $\sim 10$ min before each task and $\sim 1 \mathrm{~h}$ apart. Participants completed two reward measures; the revised monetary incentive delay (MID) (MID.R) was completed first and is reported here (see Rose et al (2014) for other outcomes). To match experimental conditions, physiological measures (eg, EKG, blood pressure, pulse oximetry; were obtained for CD and $\mathrm{HC}$ during scanning. While heart rate and pulse oximetry were obtained continually throughout scanning, blood pressure readings were paused during the functional tasks, so as not to interfere with responding or task performance. HC did not receive intravenous saline or cocaine in either session.

\section{MID.R}

The MID.R (Figure 1) has been described previously (Rose et al, 2013). Briefly, participants responded to a target (ie, white cross) with a button press during its visual presentation. To approximate a 'success'/ 'hit' rate of $2 / 3$, the target duration was varied 'online' in $25 \mathrm{~ms}$ increments, depending on the preceding success rate. Pretarget, participants viewed prime-1, which indicated trial valence (gain, lose, neutral), and prime-2, which indicated trial magnitude (small, medium, and large). A hit on gain trials $(N=85)$ resulted in a monetary gain equivalent to the prime- 2 value (US\$2, 50, 10, or 15), whereas only US\$1 was gained for a 'miss'. On loss trials $(N=85)$, a hit incurred a US $\$ 0.75$ loss, whereas a miss resulted in loss equal to prime-2 magnitude (US\$1.50, 6, and 9). Participants neither won nor lost on neutral trials $(N=28)$. The trial concluded with feedback, showing the trial outcome and current monetary total. Trials were presented across 4, 10 min runs, which included variable rest periods $(N=64)$, to add temporal jitter. Participants received a bonus payment equaling $10 \%$ of their total winnings (US $\$ 50$ per session). Withdrawal measures were not obtained during scanning, but CD used visual analog scales to feelings of 'high', 'craving,' and 'satisfaction' that may serve as proxies for withdrawal. Ratings were obtained at the start of scanning and at the beginning, middle, and end of each reward measure. Although outcomes for the second reward measure are not reported here, analysis of affective ratings during the temporal difference error (TDE) task were indicative of a similar pattern of ratings across the task as were seen here for the MID.R (see Rose et al, 2014).

\section{Functional Imaging}

Whole-brain (WB) echo planar images were acquired on a 3T Siemens Allegra scanner (Erlangen, Germany). Oblique axial slices $\left(39 \times 4 \mathrm{~mm}^{2} ; 30^{\circ} \mathrm{AC}-\mathrm{PC}\right)$ were acquired $\left(\mathrm{TR}=2000 \mathrm{~ms} ; \quad \mathrm{TE}=27 \mathrm{~ms} ; \quad \mathrm{FOV}=220 \times 220 \mathrm{~mm}^{2} \quad\right.$ at
$64 \times 64$; flip angle $\left.=78^{\circ}\right)$. T1-weighted MPRAGE structural images were also acquired (voxel $=1 \mathrm{~mm}^{3}$ ).

\section{Cocaine Metabolism}

Logistical and safety considerations precluded blood sampling during scanning. Instead, samples were collected during the cocaine toleration session in the mock scanner. This session involved $\mathrm{CD}$ receiving acute cocaine in a manner that mimicked the scanning drug administration and the task timing (see Supplementary Methods, p 2-3). Plasma samples obtained during this session were analyzed for cocaine and metabolites using liquid chromatographytandem mass spectrometry for cocaine (Figure 1c), benzoylecgonine (BE), ecgonine methyl ester (EME), and norcocaine (Lin et al, 2001, 2003).

\section{Data Analysis}

Functional imaging data were analyzed using AFNI (Cox, 1996). Data quality control procedures were as described previously (Rose et al, 2013). Data time series were analyzed using voxel-wise, multiple regressions. Regressors were expressed as a delta function series time locked to event onset and convolved with a hemodynamic response function and its temporal derivative. Regressors of interest included trial valence, magnitude, and outcome. To avoid issues of collinearity, separate analyses were conducted for each stimulus type. To account for stimulus-specific activation that was not of interest here (eg, attention, visual processing), valence analyses used contrasts of gain or loss to neutral. Due to insufficient power to model all nine feedback levels, outcome analyses considered successful (maximum gain/ minimum loss) vs unsuccessful (minimum gain/maximum loss) outcomes. This functional distinction between categories mimicked behavioral responding (ie, hit $v s$ miss) and was supported by previous analyses modeling all outcomes (Rose et al, 2013) (see Supplementary Methods for details). Voxel-wise average response amplitude (ie, percentage signal change from baseline) was calculated for each event type, participant, and session. Resultant activation maps were registered to a higher resolution $(1 \mu \mathrm{l})$ standard space (Talairach and Tournoux, 1988) and spatially blurred using a $4.2 \mathrm{~mm}$ FWHM Gaussian isotropic kernel.

Random-effects analyses considered group (ie, HC vs CD +saline $\left(\mathrm{H}_{1}\right)$ or $\mathrm{CD}+$ cocaine $\left.\left(\mathrm{H}_{2 \mathrm{~B}}\right)\right)$ and drug condition (ie, $\mathrm{CD}+$ cocaine vs $\mathrm{CD}+$ saline $\left(\mathrm{H}_{2 \mathrm{~A}}\right)$ ) effects. An analysis of session ( 1 vs 2 ) effects was performed for HC only. To assess cocaine-mediated variability outside and within classic reward pathways, WB analyses and a priori small volume correction (SVC) analyses in hypothesized DA pathway regions were performed. Bilateral regions-of-interest (ROI) were defined (Talairach and Tournoux, 1988) in the habenula, SN, striatum (nucleus accumbens, caudate, and putamen), mPFC (BA10 and BA32), and VTA. Correction for multiple comparisons was estimated for both $\mathrm{WB}$ and SVC outcomes. Significance was determined for minimum cluster extent criteria at $p_{\text {CORRECTED }} \leqslant 0.05$ and calculated separately for WB and SVC analyses using Monte Carlo simulations. For SVC analyses corrections accounted for the entire ROI/SVC volume. Outcome directionality was confirmed using corrected, predefined contrasts. 
a

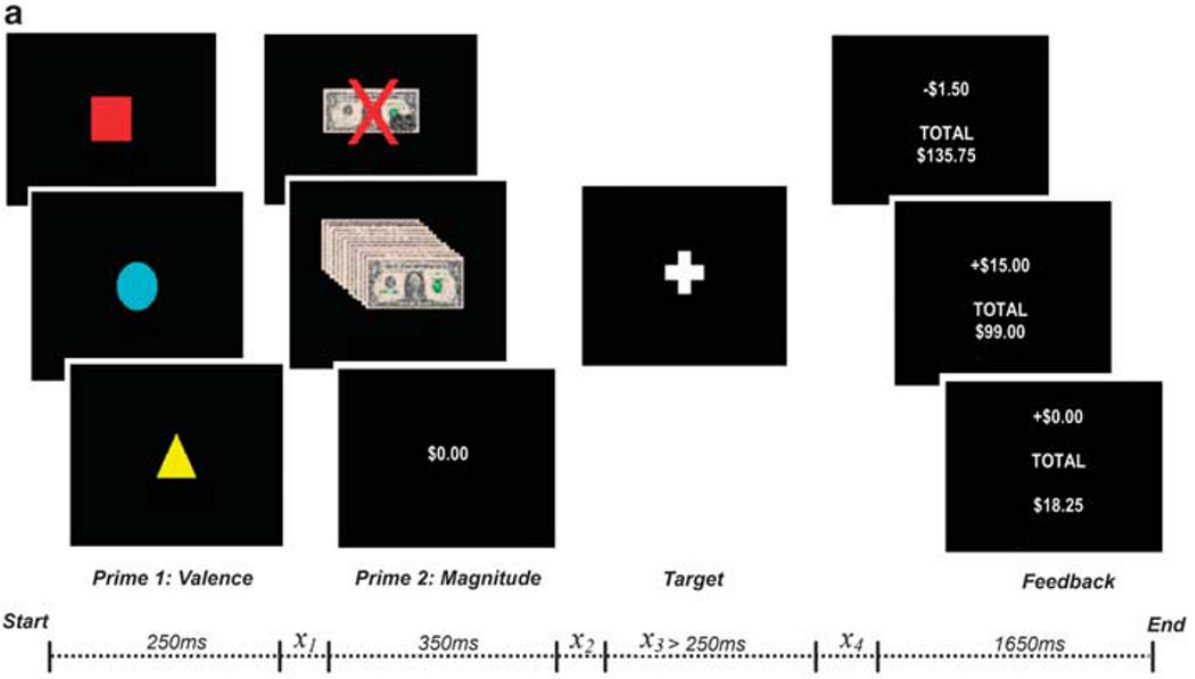

b

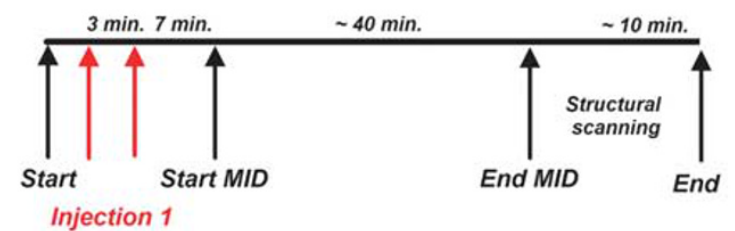

C

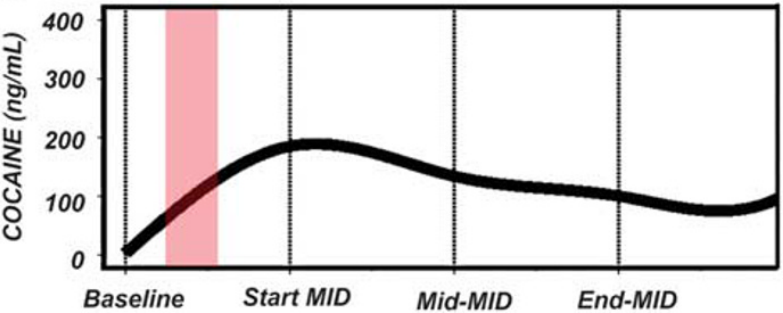

Figure I Experimental paradigm and timeline. (a) Revised monetary incentive delay (MID.R) paradigm. Note: $x$ denotes a variable presentation time, where $x_{1}$ and $x_{2}$ sum to $4000 \mathrm{~ms} ; x_{4}$ is a wait period with duration equal to the difference between $x_{3}$ and 250 ms. (b) Timing of cocaine injection relative to MID.R performance. (c) Plasma levels of cocaine $(\mathrm{ng} / \mathrm{ml})$ during practice performance of the MID.R.

In CD only, post hoc linear regressions were used to examine the relationship between chronic and acute cocaine use factors and drug-related affective states and function in regions showing an effect of group or condition. These models included mean task-related function only for those regions where group and/or condition effects had been noted in primary analyses. We also considered the relationship between activity in $\mathrm{CD}$ only in these clusters and characterization measures that differed between groups and, given our prior findings relating to nicotine-mediated effects on MID.R brain function (Rose et al, 2013), nicotine use. These post hoc analyses (ie, for nicotine and characterization measures) revealed no effects that were relevant to the current outcomes or their interpretation and thus are not reported here (see Supplementary results for full details).

\section{RESULTS}

\section{Affective Ratings}

Pre-session high and satisfied ratings did not differ between conditions $(p>0.05)$ (Supplementary Table S3). However, craving was lower preinjection in $\mathrm{CD}+$ cocaine vs $\mathrm{CD}+$ saline $\left(\mathrm{t}_{(14)}=-2.32, \quad p<0.05\right)$. High ratings were greater $\left(t_{(14)}=2.49, p<0.05\right)$ and craving was lower $\left(t_{(14)}=-2.15\right.$, $p<0.05)$ at the start of the MID.R (ie, 10 min after injection) in $\mathrm{CD}+$ cocaine $v s \mathrm{CD}+$ saline. There were no other condition-specific differences in cocaine-related ratings.

\section{MID.R: Behavioral Results}

Total winnings (US\$) and average reaction time (ms) did not differ between-condition, -group, -session (HC only) or stimulus valence and magnitude (RT only) ( $p>0.05)$ (Supplementary Table S4).

\section{Poststudy Cocaine Use}

Participants were contacted via telephone $\sim 2$ weeks after the study to complete cocaine use measures (see Supplementary Table S5 for details). No measure of cocaine consumption indicated increased use since participation, but there was a significant decrease in the duration of last cocaine binge (ie, $t_{(11)}=1.07, p<0.05$ ). 


\section{Cocaine Metabolism}

Cocaine levels peaked following cocaine and declined across the task (Figure 1c; Supplementary Table S6). Metabolite levels increased linearly across the session $(p<0.001)$ and were greater before injection 2 vs 1 (cocaine: $t_{(21)}=14.49$; BE: $t_{(21)}=15.13$; and EME: $\left.t_{(21)}=11.87 p<0.001\right)$, suggesting an accumulative effect.

\section{MID.R: Functional Imaging Results}

See Supplementary Table S7 for a detailed summary of all significant MRI results.

\section{TASK-DEPENDENT ACTIVITY (ACROSS ANALYSES)}

MID.R task-dependent activity in HC was qualitatively consistent with previous implementations of this task (Rose et al, 2013) and a similar variant of the MID used by our group (Fedota et al, 2015). Post hoc intraclass correlation analyses indicated a high level of consistency in task maps for $\mathrm{HC}$ in clusters showing a significant effect of valence, magnitude, or outcome across sessions, for both $\mathrm{WB}$ and SVC analyses (ie, in all cases $p<0.001$ ), suggesting a reasonable degree of reliability.

\section{Valence}

Valence-dependent changes in activity (ie, gain $>$ loss) were seen across WB and SVC analyses in typical reward regions, including right $(\mathrm{R})$ caudate head and left $(\mathrm{L})$ caudate body, and R-putamen, as well as outside traditional reward networks, eg, R-inferior frontal gyrus (IFG)/BA45, R-superior occipital gyrus/BA19, L-cuneus/BA17, and L-precuneus/BA7.

\section{Magnitude}

Gain magnitude. Gain magnitude also impacted function in diverse regions, including typical reward regions (ie, bilateral caudate, cingulate (BA24 and 32) and medial frontal gyrus (MFG; BA8, 10, and 32) and L-putamen) and others (ie, bilateral cerebellum and lingual gyrus (BA18), L-inferior parietal lobe (IPL)/BA40, R-cuneus (BA17, 19, and 30), R-IFG (BA46 and 47), insula/BA13, R-middle frontal gyrus/BA6, R-middle occipital gyrus/BA19, R-precentral gyrus (BA6 and 44), R-superior frontal gyrus (SFG)/BA6 and R-supramarginal gyrus/BA40). With the exception of the R-cuneus/BA17, where gain magnitude had a linear effect across all reward levels, magnitude-related activity in all other regions demonstrated a ceiling effect, with no difference between activity for medium and large magnitudes, which were both greater than activity for small anticipated rewards.

Loss magnitude. Loss magnitude influenced activity in a more restricted set of regions than gain magnitude, including in reward (ie, caudate (bilateral) and R-putamen) and other regions (ie, L-superior parietal lobe (SPL)/BA7, R-IPL/BA40, and R-middle frontal, parahippocampal, precentral, and postcentral gyri). These effects were driven by less activity for small vs medium and/or large anticipated losses. HC-only analyses noted relatively lower activity for medium $v s$ small and large loss values.

\section{Outcome}

As with anticipatory processes, outcome impacted function in reward (ie, ACC/BA32, caudate, putamen, IFG, and MFG) and other regions (ie, cingulate gyrus/BA31, claustrum, hippocampus, precuneus, SFG/BA10, and SPL/BA7). In all, activity was greater for successful $v s$ unsuccessful outcomes.

\section{$\mathrm{H}_{1}$ : $\mathrm{HC}$ vs $\mathrm{CD}+$ saline}

\section{Valence}

There were no significant effects of group (HC vs CD+saline) on valence-related activity.

\section{Magnitude}

For gain magnitude, compared with controls, CD+saline exhibited lower activity in R-putamen across gain levels (Figure 2b). Loss-magnitude activity in L-SFG/BA6 across levels was greater in CD+saline vs HC (Figure 2d).

\section{Outcome}

Although SVC analyses revealed an apparent main effect of group (ie, $\mathrm{HC}>\mathrm{CD}+$ saline) for successful and unsuccessful outcomes; Figure 3c) in the right habenula, in an adjacent and overlapping cluster this effect was specific to successful outcomes (Figure 3d), suggesting that group effects were primarily driven by a difference in successful outcomes.

Collectively, these outcomes suggest that, in line with our primary hypothesis $\left(\mathrm{H}_{1}\right)$, acutely abstinent $\mathrm{CD}$ experience a relative reduction in activity related to the anticipation and receipt of non-drug rewards. Moreover, in line with our previous results, acute abstinence was also assocaited with an increased sensitivity to anticipation of negative outcomes.

\section{$\mathrm{H}_{2 \mathrm{~A}}$ : $\mathrm{CD}+$ saline vs $\mathrm{CD}+$ cocaine}

\section{Valence}

There were no significant effects of condition (CD+saline vs $\mathrm{CD}+$ cocaine) on valence-related activity.

\section{Magnitude}

There were no significant effects of condition (CD+saline $v s$ $\mathrm{CD}+$ cocaine) on magnitude-related activity, for gains or losses.

\section{Outcome}

WB and SVC were indicative of condition-dependent variability in outcome-related activity in a region consistent with the right habenula. In this area, success-dependent activity was greater in $\mathrm{CD}+$ cocaine vs $\mathrm{CD}+$ saline (Figures $3 \mathrm{a}$ and $\mathrm{b}$ )

With respect to our second hypothesis $\left(\mathrm{H}_{2 \mathrm{~A}}\right)$, these outcomes are indicative of a differential effect of acute cocaine on underlying reward processing deficits, with cocaine effectively ameliorating outcome-dependent changes (ie, 'liking') but having no discernible impact on anticipation (ie, 'wanting'). 
$\mathrm{H}_{2 \mathrm{~B}}: \mathrm{CD}+$ cocaine $v s \mathrm{HC}$

\section{Valence}

There were no significant effects of group (HC vs CD +cocaine) on valence-related activity.

\section{Magnitude}

For gain magnitude, $\mathrm{CD}+$ cocaine exhibited lower activity in the L-cingulate gyrus/BA32 for large gain primes compared with HC (Figure 2a) while activity for loss magnitude in the

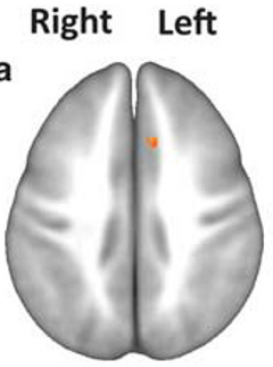

$(-92139)$

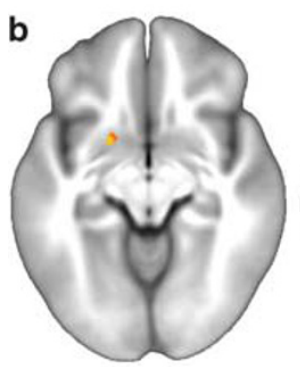

(19 $9-6)$

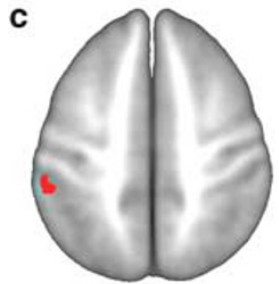

(-51 3546$)$

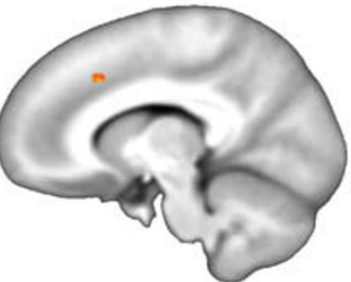

1.0

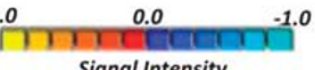

Signal Intensity
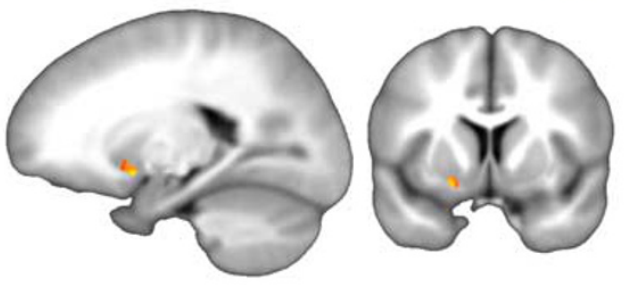

$\square$ small
$\square$ medium
$\square$ large

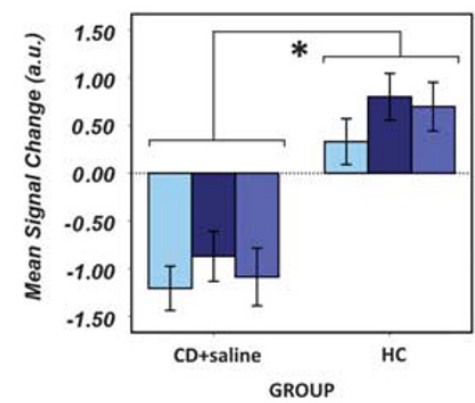

GROUP

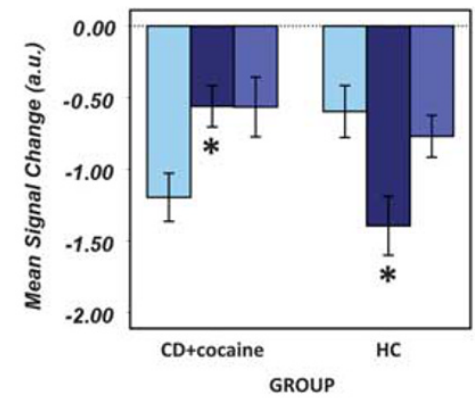

large

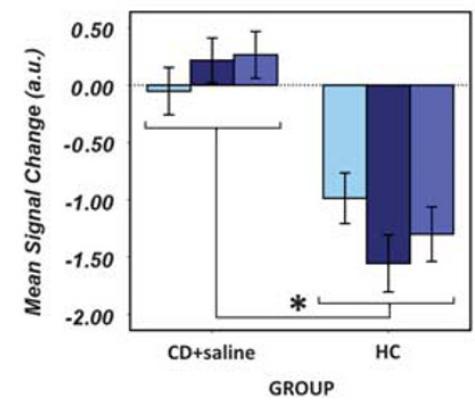

Figure 2 Group-dependent effects on magnitude-related activity. (a) Interaction between group (HC vs CD+cocaine) and gain magnitude (left cingulate gyrus/BA32; SVC analysis; cluster extent $\left.\left(K_{E}\right)=47\right)$. (b) Main effect of group (HC vs CD+saline) on gain magnitude-related activity (left putamen; SVC analysis; $K_{E}=5 \mathrm{I}$ ). (c) Interaction between group (HC vs CD+cocaine) and loss magnitude (right inferior parietal lobe/BA40; WB analysis; $K_{E}=238$ ). (d) Main effect of group (HC vs CD+saline) on loss of magnitude-related activity (left superior frontal gyrus; WB analysis; $K_{E}=283$ ). Note: * PCORRECTED <0.05. Error bars show \pm I SE; activation maps are rendered on the ICBM452 TI template from AFNl; right = left; minimum corrected $K_{E}=200$ for WB and 47 for SVC analyses; see Supplementary Figures S4A and B for task-dependent activity distributions corresponding to these significant effects. 

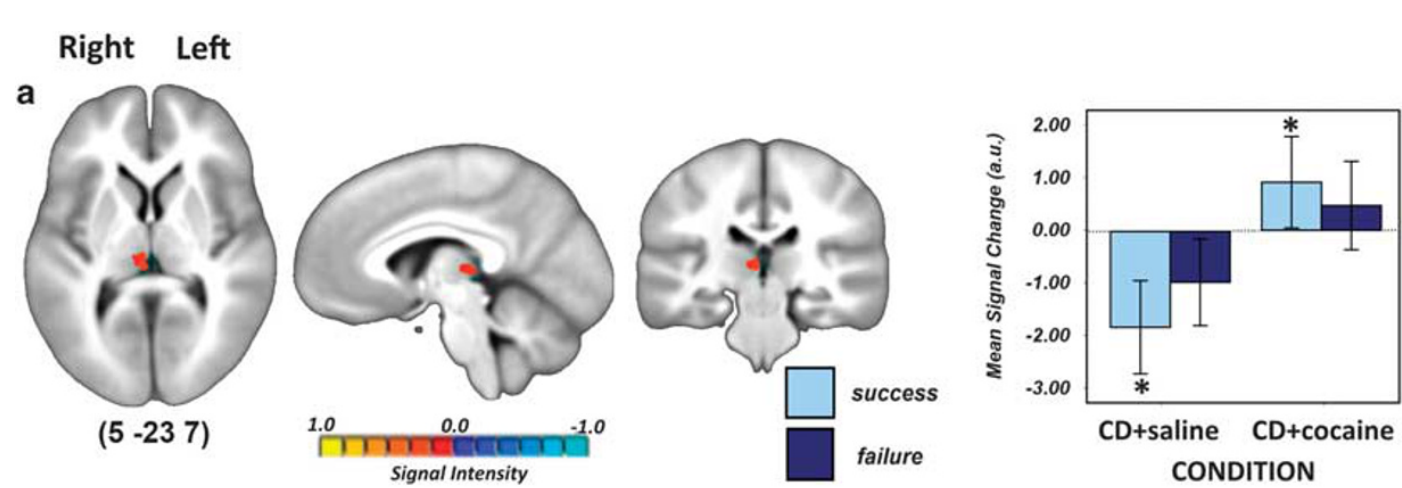

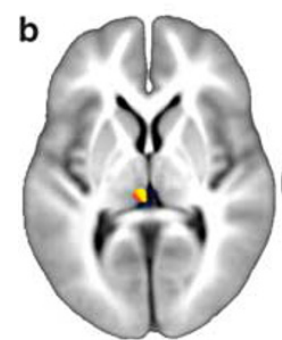

$(5-237)$
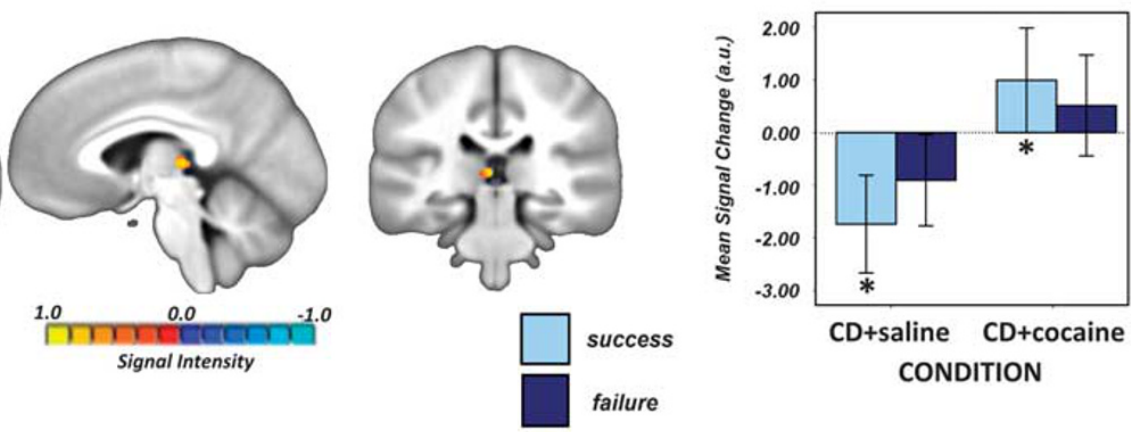

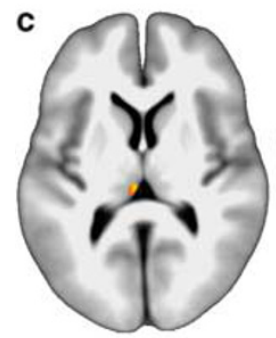

$(6-249)$
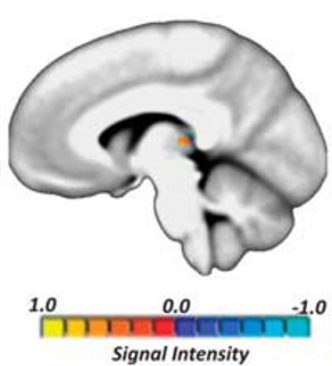

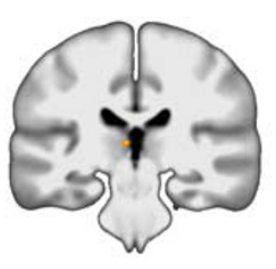

$\square$ success
$\square$ failure

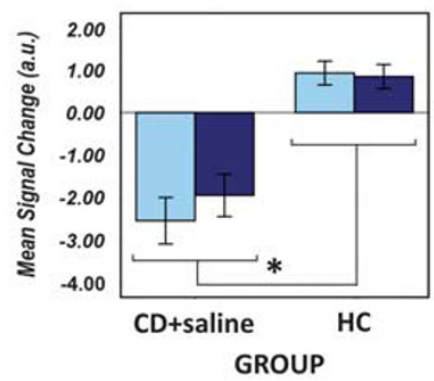

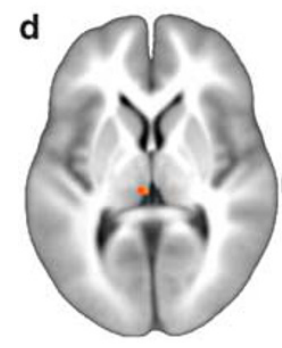

$(5-237)$
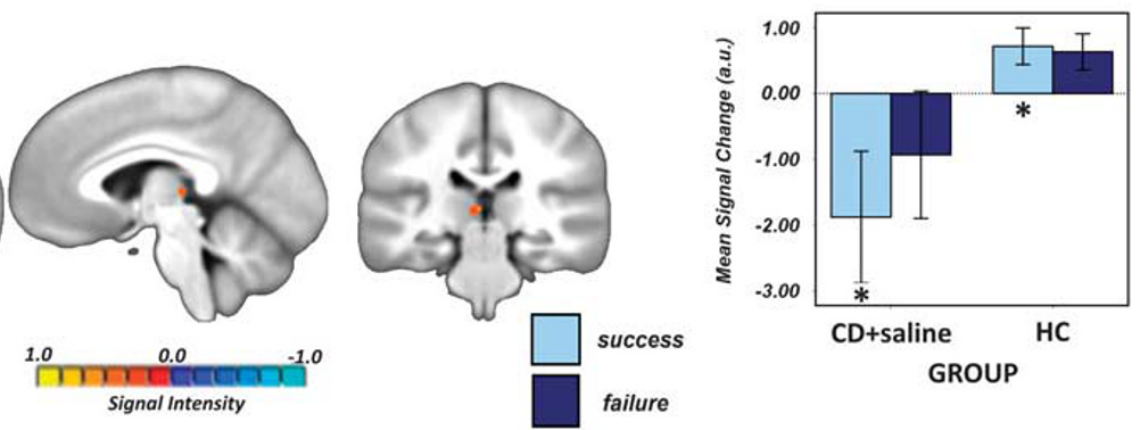

Figure 3 Group- and condition-dependent effects on outcome-related activity in the right habenula. (a) condition $\times$ outcome interaction (WB analysis; $K_{E}=223$ ); (b) condition $\times$ outcome interaction (SVC analysis; $K_{E}=177$ ); (c) Main effect of group (HC vS CD+saline; SVC analysis; $K_{E}=5 \mathrm{I}$ ); (d) group ( $\mathrm{HC}$ vs CD+saline) $\times$ outcome interaction (SVC analysis; $K_{E}=67$ ). Note: * $=$ PCORRECTED $<0.05$. Error bars show \pm I SE; activation maps are rendered on the ICBM452 TI template from AFNI; right= left; minimum corrected $K_{E}=200$ for WB and 47 for SVC; see Supplementary Figure S4C for task-dependent activity distributions corresponding to these significant effects.

R-IPL/BA40 was greater for medium magnitude stimuli in $\mathrm{CD}+$ cocaine $v s \mathrm{HC}$ (Figure 2c).

\section{Outcome}

There were no significant effects of group (HC vs CD +cocaine) on outcome-related activity.
These outcomes partially support our second hypothesis $\left(\mathrm{H}_{2 \mathrm{~B}}\right)$, in that there were no observed differences between $\mathrm{HC}$ and $\mathrm{CD}+$ saline in the same region (habenula) where effects of group (HC vs $\mathrm{CD}+$ saline) and condition were noted in other analyses. However, there were distinctions between $\mathrm{HC}$ and $\mathrm{CD}+$ cocaine in anticipatory function for magnitude, 

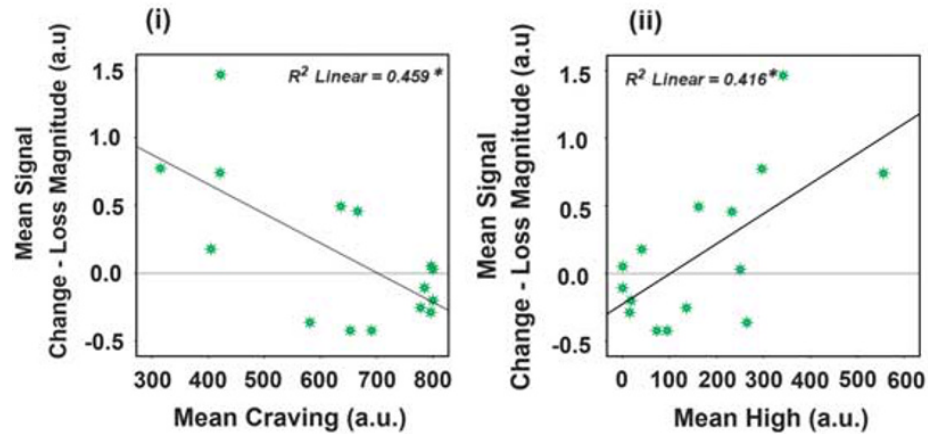

\section{$\Delta \mathrm{CD}+$ cocaine * CD+saline}

Right habenula

(i)

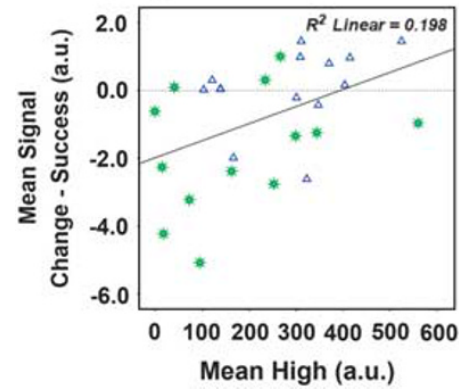

(ii)

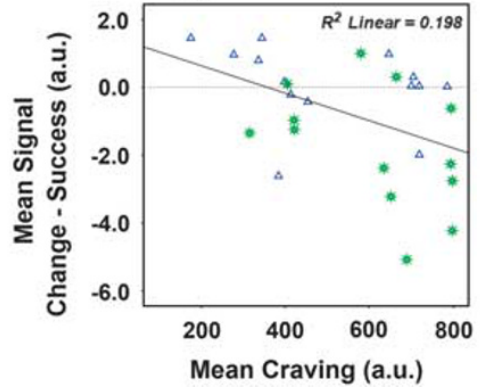

(iii)

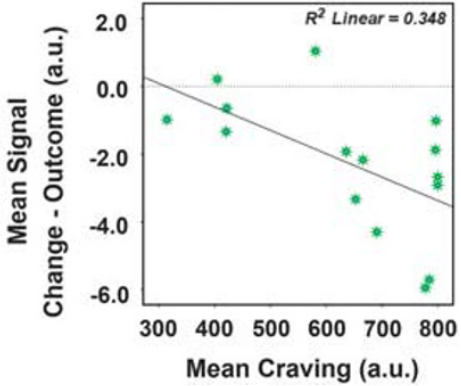

Figure 4 Correlations between group- and condition-dependent variability in brain function and affective ratings. Significant associations with activity are shown for: (a) group-dependent differences in magnitude-related activity in the left superior frontal gyrus/BA6 for CD+saline only and (i) craving and (ii) high (WB analysis); and (b) outcome-related differences in function in the right habenula for success-related activity in CD+cocaine and CD+saline and ratings of (i) high and (ii) craving (WB analysis) and across outcomes for CD+saline only and (iii) craving (SVC analysis). Note: All $p<0.05$; *PCORRECTED $<0.0$ I25.

suggesting that any 'normalization' of reward function after cocaine administration may be limited.

\section{Post hoc Connectivity Analysis}

Since reciprocal relationships between the habenula and other reward pathway regions modulate motivational responding (Hikosaka et al, 2008), we conducted post hoc connectivity analyses using the putative habenula cluster as a seed (methods as described in Sutherland et al, 2013). Activity in this cluster was highly positively correlated with all other SVC regions including VTA $\left(p_{\text {corrected }}<0.05\right)$. However, there was no group- or condition-dependent variability in the extent or direction of habenular connectivity.

\section{Post hoc Linear Regressions}

These CD-only regression analyses considered associations between activity in regions where we found group and/or condition effects and affective ratings (ie, high, craving, satisfied) and indices of chronic (ie, age at first use and years of use) and recent use (ie, days used cocaine, rocks used and spend (\$) on cocaine in the week prestudy and time (days) between last binge and study entry).

While use indices did not predict group- or conditiondependent differences, functional variability was predicted by 'craving' and 'high' ratings. In the L-SFG/BA6, magnituderelated activity on loss trials correlated negatively with 'craving' $\left(\mathrm{F}_{(1,14)}=11.01, p<0.05\right.$; Figure $\left.4 \mathrm{a}(\mathrm{i})\right)$ and positively with 'high' ratings $\left(\mathrm{F}_{(1,14)}=9.28, p<0.05\right.$; Figure 4aii; note: these results survived Bonferroni correction for multiple comparisons for the number of regions considered, ie, $N=4$ ). These ratings were also predictive of success-related activity in R-habenula across conditions (craving: WB: $\mathrm{F}_{(1,26)}=6.87$ and SVC: $\mathrm{F}_{(1,26)}=6.54$; high: WB: $\mathrm{F}_{(1,26)}=5.49$ and SVC: $\left.\mathrm{F}_{(1,26)}=5.77 ; \quad p<0.05\right)$. As 'craving' increased, habenula activity decreased, whereas the opposite was true for 'high' ratings (Figure $4 \mathrm{~b}(\mathrm{i})$ and (ii)). In the R-habenula cluster where we observed differences between $\mathrm{CD}$ +saline and $\mathrm{HC}$ outcome-related activity for $\mathrm{CD}$ also negatively correlated with craving $\left(\mathrm{F}_{(1,14)}=6.95, p<0.05\right.$; Figure $\left.4 \mathrm{~b}(\mathrm{iii})\right)$.

\section{DISCUSSION}

We interrogated the impact of CUD on distinct reward processes in the presence and absence of acute cocaine. Group and condition did not impact task performance or valence-dependent activity. However, contradictory to studies showing CD-specific effects on incentive processing (Jia et al, 2011; Patel et al, 2013), our MID.R task is unique in dissociating the valence and magnitude components of anticipatory stimuli. It is perhaps this dissociation that is driving this difference. That is, cocaine-dependent variability 
in incentive processing may not simply be related to valence but also other contextual information.

\section{The Impact of Cocaine on Anticipatory Brain Activity}

Consistent with this postulation, group- and conditionspecific effects were noted for magnitude-dependent anticipatory processing. Across conditions, CD were less sensitive to impending rewards than $\mathrm{HC}$ in typical MCL rewardrelated regions, showing reduced activity in BA32/dorsal ACC for large gains in the $\mathrm{CD}+$ cocaine condition and the putamen for all gain values in the abstinence condition (ie, saline). In contrast, CD were more sensitive to impending negative outcomes than $\mathrm{HC}$, across conditions, in areas typically linked to motor processes. For example, CD +cocaine showed reduced activity for medium losses in an IPL region associated with motor planning and action (ie, rostral IPL/BA40; Caspers et al, 2010, 2013), whereas loss-related function was reduced in CD+saline in L-SFG/ BA6. Thus, motivational deficits in $\mathrm{CD}$ may be attributable to reduced sensitivity to anticipated rewards in reward networks and increased sensitivity to impending punishing outcomes, which manifests via planned actions (ie, motor responding) and the regions that mediate these functions.

Increased sensitivity to impending negative outcomes and decreased sensitivity to upcoming rewards in CD here aligns with previous work in the same cohort (Rose et al, 2014). It is intriguing that the pattern of regions implicated in functional alterations to positive and negative reinforcing outcomes in that TDE analysis were highly similar to those noted here for altered magnitude-dependent function. For example, positive TDE signal alterations in CD involved regions within DA reward networks, including the putamen and BA32, whereas differences in negative TDE processing in $\mathrm{CD}$ were noted in non-reward regions, including premotor cortex/BA6. Collectively, outcomes across measures suggest a distinction in regions underlying the differential impact of CUD on positive and negative reinforcers. They also indicate that the impact of CUD in these regions is nonspecific for reward type (primary $v s$ secondary) and can be observed at distinct reward processing stages. Thus, sensitivity to negative outcomes and insensitivity to positive ones may persist across different aspects of individual's daily lives. This may be relevant to clinical outcomes for $\mathrm{CD}$ via consequences for decision-making, particularly in relation to drug use decisions.

Although magnitude-dependent effects were limited in CD +cocaine, they were more generalized during acute abstinence. This suggests a partial amelioration of such effects after cocaine administration. In line with the 'self-medication' hypothesis (Khantzian, 1985), this indicates that a potential consequence of cocaine administration is an adjustment in incentive processing that reduces dependence-related deficits. This is in keeping with studies demonstrating a reduction in the extent of reward processing deficits in $\mathrm{CD}$ with cocaine positive $v s$ negative urine toxicology (Parvaz et al, 2012). The lack of specificity in dependence-related effects in CD+saline is also in line with the others showing a lack of sensitivity to monetary gradients in CD (Goldstein et al, 2007a, b, 2008; Konova et al, 2012). However, while Goldstein and co-workers found mostly flat magnitude effects in current users, our $\mathrm{CD}+$ cocaine group showed variable sensitivity to gain and loss magnitude. This may be the consequence of more recent cocaine use in our group. If use recency predicts the extent of dependence-related deficits, it is unsurprising that monitoring reward function immediately after cocaine administration would result in a pattern of brain function not entirely like that observed in individuals whose use likely occurred many hours pretesting. In this respect, our $\mathrm{CD}+$ saline group is actually more like those who tested positive in the Goldstein studies. Importantly, we did not observe a similar effect of acute cocaine administration for our TDE paradigm, suggesting that such effects may be stimulusspecific and perhaps limited to cocaine-associated stimuli, for example, money. Potential monetary gains and losses may have very specific connotations for CD (eg, money influences the ability to obtain cocaine), and this relevance may vary depending on whether $\mathrm{CD}$ are high or abstinent and craving cocaine.

\section{The Impact of Cocaine on the Hedonic Experience of Non-Drug Rewards}

While the TDE analyses revealed group-specific effects on outcome-related function (Rose et al, 2014), here we found group- and condition-specific effects in a region consistent with the habenula. In this cluster success-related activity was reduced in $\mathrm{CD}+$ saline vs $\mathrm{CD}+$ cocaine condition and $\mathrm{HC}$. We should be cautious in our interpretation of this outcome because of difficulties modeling habenula function in human MRI studies (eg, due to the small size of the habenula relative to voxel size and partial-voluming concerns). Moreover, it should be considered in the context of a lack of corresponding effects in more typical reward areas. Nonetheless, it is intriguing that relatively consistent abstinence-dependent effects on outcome-related activity in CD were noted in this region, as it is positioned to mediate affective behaviors relevant to motivation in healthy and clinical populations and the brain's response to cocaine.

The habenula consists of subnuclei that form medial and lateral divisions (Andres et al, 1999), which are innervated by afferents from limbic regions (ie, medial) and basal ganglia (ie, lateral). LHb efferents descend primarily to the brainstem and target monoamingeric neurons, including the DArgic VTA and SN (Hikosaka et al, 2008). The LHb contributes to the control of cognitive and motor behaviors by manipulating motivational value (Hikosaka et al, 2008). Preclinical studies suggest that rewarding/successful outcomes decrease $\mathrm{LHb}$ activity and a concomitant increase in VTA activity and striatal DA release, driving the organism to repeat the behavior. In contrast, punishing/unsuccessful outcomes increase LHb activity resulting in decreased VTA activity and striatal DA release and, ultimately, avoidance.

The LHb is also a target for cocaine (Zhang et al, 2013). Preclinical electrophysiological models show that cocaine induces a biphasic effect on LHb neurons (Jhou et al, 2013), ie, initial inactivation of $\mathrm{LHb}$ neurons immediately following cocaine administration, followed by increased activity $\sim 15$ min after injection. Thus, cocaine may be rewarding or punishing depending on the time of assessment relative to administration, which has implications for behavioral measures after drug administration. 
Human studies implicate the LHb in processing aversive or punishing signals, such as error detection (Li et al, 2008) or aversive electric shock (Hennigan et al, 2015; Lawson et al, 2014). Such studies also demonstrate a feed-forward influence of the habenula on VTA/SN activity (Ide and Li, 2011). Moreover, in line with preclinical observations (Danna et al, 2013), habenula function in humans is implicated in drug addiction (Salas et al, 2010; Velasquez et al, 2014) monetary reward (Lawson et al, 2014), and negative TDE signals (Salas et al, 2010).

Here there was no main effect of valence, magnitude, or outcome on habenular activity. Rather habenular effects were specific to experiencing reinforcing outcomes during acute cocaine abstinence. As LHb activity is associated with punishing/aversive outcomes, our observations suggest habenula activity in abstinent $\mathrm{CD}$ was more typical of what one would expect to see for rewarding outcomes (ie, it decreased), whereas in $\mathrm{HC}$ and $\mathrm{CD}+$ cocaine, habenula activity was more typically 'aversive' (ie, increased). However, given the positive correlation between the habenula and VTA, which is in keeping with others (Hennigan et al, 2015) and the extended time between cocaine use $(>24 \mathrm{~h})$ and measurement, decreases in habenula in $\mathrm{CD}+$ saline may have related to a functional cascade more typical of punishing/aversive outcomes. That is, at the time we measured reward function, $\mathrm{CD}+$ saline were perhaps entering a phase of habenular responding where perceptions are more aversive. Although plausible, preclinical studies have not tracked $\mathrm{LHb}$ response after cocaine administration for a duration that would approximate the time between last cocaine use and imaging in $\mathrm{CD}+$ saline, making this interpretation speculative at best. Although exploration in other models is necessary, our data preliminarily suggest that post-cocaine changes in LHb function may persist for extended periods and are characterized by a pattern of responding that does not align with the current short-term opponent process model of cocaine-mediated effects on $\mathrm{LHb}$ function.

\section{The Role of the Affective Sequelae of Cocaine Use in Non-Drug Reward Processing}

Although group- and condition-dependent effects on function in CD were not predicted by indices of cocaine use, they were associated with ratings of high and craving. Thus, cocaine-dependent variability in reward processing is not only predicted by CUD and/or acute intoxication but also by the extent of craving. This is potentially relevant for cocaine seeking and continued use during attempted abstinence and so may be important for treatment. For example, if acute cocaine engenders more normalized responding to non-drug reinforcers and reduced craving, the drive to consume cocaine may be related not specifically to the experience of 'high' but rather the facilitation of more normative rewarding experiences in general. Contrastingly, a reduced ability to respond appropriately to non-drug reinforcers while abstinent, which is exaggerated by cocaine craving, is a potentially powerful driving force for continued use. In line with this, reward processing abnormalities in CD have been shown to be important for predicting cessation (Stewart et al, 2014).

\section{Study Limitations}

A limitation here is the relatively small number of subjects for a neuroimaging investigation. Although this concern speaks to the need for replication in larger samples, we are nevertheless confident of the reliability of the outcomes noted here given our conservative approach to data quality control and conservative correction methods in our statistical analysis. Moreover, the task maps for the MID.R are highly consistent with those seen by our group with other larger cohorts (eg, Fedota et al, 2015; Rose et al, 2013).

\section{CONCLUSIONS}

Across studies our data suggest that the trait-like experience of CUD and the state of acute cocaine intoxication differentially impact distinct reward processes. Such effects are related to situational factors such as craving, and can be seen across reward types. These patterns may be relevant for continued cocaine use and treatment outcomes. In particular, variability in reward function may be a contributory factor to individual differences in treatment outcome, and in particular for those studies that rely on reinforcement (eg, contingency management). The impact of treatment on reward processing may be a crucial measure of treatment efficacy and developing effective treatments for CUD may be advanced by a translational approach to the delineation of motivational deficit variability in $\mathrm{CD}$, understanding how this mediates outcomes and determining the neural mechanisms by which this occurs. In addition to evaluating psychotherapeutic interventions, our paradigm may prove useful for understanding alternative therapeutic approaches and determining who is more likely to respond. For example, it has been suggested that the LHb is a suitable target for substance dependence in general (Yadid et al, 2013) and for cocaine use specifically (Alba-Ferrara et al, 2014). Use of a similar paradigm may help determine variability in baseline/ pretreatment function that could serve as a response biomarker, perhaps also including predicting individuals at risk for drug dependence.

\section{FUNDING AND DISCLOSURE}

The authors declare no conflict of interest.

\section{ACKNOWLEDGMENTS}

We gratefully acknowledge Kimberley Slater for her invaluable support and assistance in conducting this study. We also thank NIDA-IRP nursing, recruitment and support staff, and all those individuals who volunteered their time to participate. This work was supported by the National Institute on Drug Abuse-Intramural Research Program.

\section{REFERENCES}

Alba-Ferrara LM, Fernandez F, de Erausquin GA (2014). The use of neuromodulation in the treatment of cocaine dependence. Addict Disord Their Treat 13: 1-7.

Andres KH, von During M, Veh RW (1999). Subnuclear organization of the rat habenular complexes. J Comp Neurol 407: $130-150$. 
Asaad WF, Eskandar EN (2011). Encoding of both positive and negative reward prediction errors by neurons of the primate lateral prefrontal cortex and caudate nucleus. J Neurosci 31: 17772-17787.

Berns GS, McClure SM, Pagnoni G, Montague PR (2001). Predictability modulates human brain response to reward. J Neurosci 21: 2793-2798.

Breiter HC, Gollub RL, Weisskoff RM, Kennedy DN, Makris N, Berke JD et al (1997). Acute effects of cocaine on human brain activity and emotion. Neuron 19: 591-611.

Caspers S, Schleicher A, Bacha-Trams M, Palomero-Gallagher N, Amunts K, Zilles K (2013). Organization of the human inferior parietal lobule based on receptor architectonics. Cereb Cortex 23: 615-628.

Caspers S, Zilles K, Laird AR, Eickhoff SB (2010). ALE meta-analysis of action observation and imitation in the human brain. NeuroImage 50: 1148-1167.

Cox RW (1996). AFNI software for analysis and visualization of functional magnetic resonance neuroimages. Comput Biomed Res 29: $162-173$.

Danna CL, Shepard PD, Elmer GI (2013). The habenula governs the attribution of incentive salience to reward predictive cues. Front Hum Neurosci 7: 781.

Diekhof EK, Falkai P, Gruber O (2008). Functional neuroimaging of reward processing and decision-making: a review of aberrant motivational and affective processing in addiction and mood disorders. Brain Res Rev 59: 164-184.

Fedota JR, Sutherland MT, Salmeron BJ, Ross TJ, Hong LE, Stein EA (2015). Reward anticipation is differentially modulated by varenicline and nicotine in smokers. Neuropsychopharmacology 40: 2038-2046.

Goldstein RZ, Alia-Klein N, Tomasi D, Zhang L, Cottone LA, Maloney $\mathrm{T}$ et al (2007a). Is decreased prefrontal cortical sensitivity to monetary reward associated with impaired motivation and self-control in cocaine addiction? Am J Psychiatry 164: 43-51.

Goldstein RZ, Parvaz MA, Maloney T, Alia-Klein N, Woicik PA, Telang $\mathrm{F}$ et al (2008). Compromised sensitivity to monetary reward in current cocaine users: an ERP study. Psychophysiology 45: 705-713.

Goldstein RZ, Tomasi D, Alia-Klein N, Cottone LA, Zhang L, Telang $\mathrm{F}$ et al (2007b). Subjective sensitivity to monetary gradients is associated with frontolimbic activation to reward in cocaine abusers. Drug Alcohol Depend 87: 233-240.

Gu H, Salmeron BJ, Ross TJ, Geng X, Zhan W, Stein EA et al (2010). Mesocorticolimbic circuits are impaired in chronic cocaine users as demonstrated by resting-state functional connectivity. NeuroImage 53: 593-601.

Hennigan K, D'Ardenne K, McClure SM (2015). Distinct midbrain and habenula pathways are involved in processing aversive events in humans. I Neurosci 35: 198-208.

Hikosaka O, Sesack SR, Lecourtier L, Shepard PD (2008). Habenula: crossroad between the basal ganglia and the limbic system. J Neurosci 28: 11825-11829.

Ide JS, Li CS (2011). Error-related functional connectivity of the habenula in humans. Front Hum Neurosci 5: 25.

Jhou TC, Good CH, Rowley CS, Xu SP, Wang H, Burnham NW et al (2013). Cocaine drives aversive conditioning via delayed activation of dopamine-responsive habenular and midbrain pathways. J Neurosci 33: 7501-7512.

Jia Z, Worhunsky PD, Carroll KM, Rounsaville BJ, Stevens MC, Pearlson GD et al (2011). An initial study of neural responses to monetary incentives as related to treatment outcome in cocaine dependence. Biol Psychiatry 70: 553-560.

Khantzian EJ (1985). The self-medication hypothesis of addictive disorders: focus on heroin and cocaine dependence. Am J Psychiatry 142: 1259-1264.
Knutson B, Adams CM, Fong GW, Hommer D (2001). Anticipation of increasing monetary reward selectively recruits nucleus accumbens. J Neurosci 21: RC159.

Knutson B, Fong GW, Bennett SM, Adams CM, Homme D (2003). A region of mesial prefrontal cortex tracks monetarily rewarding outcomes: characterization with rapid event-related fMRI. NeuroImage 18: 263-272.

Konova AB, Moeller SJ, Tomasi D, Parvaz MA, Alia-Klein N, Volkow ND et al (2012). Structural and behavioral correlates of abnormal encoding of money value in the sensorimotor striatum in cocaine addiction. Eur J Neurosci 36: 2979-2988.

Kufahl PR, Li Z, Risinger RC, Rainey CJ, Wu G, Bloom AS et al (2005). Neural responses to acute cocaine administration in the human brain detected by fMRI. NeuroImage 28: 904-914.

Lawson RP, Seymour B, Loh E, Lutti A, Dolan RJ, Dayan P et al (2014). The habenula encodes negative motivational value associated with primary punishment in humans. Proc Natl Acad Sci USA 111: 11858-11863.

Li CS, Yan P, Chao HH, Sinha R, Paliwal P, Constable RT et al (2008). Error-specific medial cortical and subcortical activity during the stop signal task: a functional magnetic resonance imaging study. Neuroscience 155: 1142-1151.

Lin SN, Moody DE, Bigelow GE, Foltz RL (2001). A validated liquid chromatography-atmospheric pressure chemical ionizationtandem mass spectrometry method for quantitation of cocaine and benzoylecgonine in human plasma. J Anal Toxicol 25: 497-503.

Lin SN, Walsh SL, Moody DE, Foltz RL (2003). Detection and time course of cocaine $\mathrm{N}$-oxide and other cocaine metabolites in human plasma by liquid chromatography/tandem mass spectrometry. Anal Chem 75: 4335-4340.

Matsumoto M, Hikosaka O (2008). Negative motivational control of saccadic eye movement by the lateral habenula. Prog Brain Res 171: 399-402.

McClure SM, Berns GS, Montague PR (2003). Temporal prediction errors in a passive learning task activate human striatum. Neuron 38: $339-346$.

O'Doherty J, Kringelbach ML, Rolls ET, Hornak J, Andrews C (2001). Abstract reward and punishment representations in the human orbitofrontal cortex. Nat Neurosci 4: 95-102.

Parvaz MA, Maloney T, Moeller SJ, Woicik PA, Alia-Klein N, Telang $\mathrm{F}$ et al (2012). Sensitivity to monetary reward is most severely compromised in recently abstaining cocaine addicted individuals: a cross-sectional ERP study. Psychiatry Res 203: 75-82.

Patel KT, Stevens MC, Meda SA, Muska C, Thomas AD, Potenza MN et al (2013). Robust changes in reward circuitry during reward loss in current and former cocaine users during performance of a monetary incentive delay task. Biol Psychiatry 74: $529-537$.

Risinger RC, Salmeron BJ, Ross TJ, Amen SL, Sanfilipo M, Hoffmann RG et al (2005). Neural correlates of high and craving during cocaine self-administration using BOLD fMRI. NeuroImage 26: 1097-1108.

Rose EJ, Ross TJ, Salmeron BJ, Lee M, Shakleya DM, Huestis MA et al (2013). Acute nicotine differentially impacts anticipatory valence- and magnitude-related striatal activity. Biol Psychiatry 73: $280-288$

Rose EJ, Salmeron BJ, Ross TJ, Waltz J, Schweitzer JB, McClure SM et al (2014). Temporal difference error prediction signal dysregulation in cocaine dependence. Neuropsychopharmacology 39: 1732-1742.

Salas R, Baldwin P, de Biasi M, Montague PR (2010). BOLD responses to negative reward prediction errors in human habenula. Front Hum Neurosci 4: 36.

SAMHSA (2014). The NSDUH Report: substance use and mental health estimates from the 2013 National Survey on Drug Use and 
Health: overview of findings. In: CfBHSaQ (ed). Substance Abuse and Mental Health Administration. SAMHSA: Rockville, MD.

Schultz W (1998). Predictive reward signal of dopamine neurons. J Neurophysiol 80: 1-27.

Schultz W (2000). Multiple reward signals in the brain. Nat Rev Neurosci 1: 199-207.

Stewart JL, Connolly CG, May AC, Tapert SF, Wittmann M, Paulus MP (2014). Cocaine dependent individuals with attenuated striatal activation during reinforcement learning are more susceptible to relapse. Psychiatry Res 223: 129-139.

Sutherland MT, Carroll AJ, Salmeron BJ, Ross TJ, Stein EA (2013). Insula's functional connectivity with ventromedial prefrontal cortex mediates the impact of trait alexithymia on state tobacco craving. Psychopharmacology (Berl) 228: 143-155.

Talairach J, Tournoux P (1988). Co-Planar Stereotaxic Atlas of the Human Brain. Thieme: New York, NY.

Tomasi D, Volkow ND, Wang R, Carrillo JH, Maloney T, Alia-Klein $\mathrm{N}$ et al (2010). Disrupted functional connectivity with dopaminergic midbrain in cocaine abusers. PLoS One 5: e10815.

Velasquez KM, Molfese DL, Salas R (2014). The role of the habenula in drug addiction. Front Hum Neurosci 8: 174.

Volkow ND, Wang GJ, Fowler JS, Tomasi D, Telang F (2011). Addiction: beyond dopamine reward circuitry. Proc Natl Acad Sci USA 108: 15037-15042.

Volkow ND, Wang GJ, Fowler JS, Tomasi D, Telang F, Baler R (2010). Addiction: decreased reward sensitivity and increased expectation sensitivity conspire to overwhelm the brain's control circuit. BioEssays 32: 748-755.

Yadid G, Gispan I, Lax E (2013). Lateral habenula deep brain stimulation for personalized treatment of drug addiction. Front Hum Neurosci 7: 806.

Zhang CX, Zhang H, Xu HY, Li MX, Wang S (2013). The lateral habenula is a common target of cocaine and dexamethasone. Neurosci Lett 555: 12-17.

Supplementary Information accompanies the paper on the Neuropsychopharmacology website (http://www.nature.com/npp) 